Volume 8. No. 10, October 2020

International Journal of Emerging Trends in Engineering Research

Available Online at http://www.warse.org/IJETER/static/pdf/file/ijeter098102020.pdf

https://doi.org/10.30534/ijeter/2020/098102020

\title{
Application of Homotopy Analysis Method for Investigating Nonlinear Oscillations
}

\author{
B.Mahaboob ${ }^{1}$, C.Narayana ${ }^{2}$, P.Sreehari Reddy ${ }^{3}$, M.Sivaiah ${ }^{4}$, J. Peter Praveen ${ }^{5}$, B. Nageswara Rao ${ }^{6}$ \\ ${ }^{1}$ Department of Mathematics, Koneru Lakshmaiah Education Foundation, Vaddeswaram, Guntur, India-522502, \\ Email: mahaboob@kluniversity.in \\ ${ }^{2}$ Department of Mathematics, Sri Harsha Institute of P.G Studies, Nellore, A.P, Email: nareva.nlr@ gmail.com \\ ${ }^{3}$ Department of Mathematics NBKR Science\& Arts College, Vidyanagar, Nellore, A.P, India \\ Email:sreeharireddy8969@gmail.com \\ ${ }^{4}$ Department of Mathematics, NBKR Science\& Arts College, Vidyanagar, Nellore, A.P, \\ India.Email:drmallisivaiah@gmail.com \\ ${ }^{5}$ Department of Mathematics, Koneru Lakshmaiah Education Foundation, Vaddeswaram, Guntur, India-522502, \\ Email: jppraveen17@kluniversity.in \\ ${ }^{6}$ Department of Mechanical Engineering, Koneru Lakshmaiah Education Foundation, Vaddeswaram, Guntur, \\ India-522502, Email: bnrao52@ rediffmail.com
}

\begin{abstract}
Homotopy Analysis Method (HAM) is a well organized method to get the periodic solution of Non-Linear Oscillatory Duffing Equation of Motion. By having a glance on Helmholtz Equation of Motion an attempt has been made to explore the proficiency of HAM. This research article explores on Helmholtz Equation of Motion possessing non-odd restoring force function. Moreover in this equation the behavior of oscillations is different for the same magnitude of $+v e$ and $-v e$ amplitudes. This phenomenon concerns with asymmetric oscillations which are nonlinear. In the case of large amplitude vibrations a greater amount of inconsistency has been noticed here. Furthermore the incapability of HAM in differentiating the non-periodic solution of Motion has been extensively discussed.
\end{abstract}

Key words : Homotopy Analysis Method (HAM); Duffing Equation; Helmholtz's equation of Motion (HEM) ; Periodic solution; Phase-plane diagram, Differential Equation (DE)

\section{INTRODUCTION}

A larger number of problems in Engineering Sciences are modeled by Non-Linear Duffing Equation of Motion namely

$$
\frac{d^{2} x}{d t^{2}}+g(x)=G(t)
$$

Here restoring force function which is a polynomial is given by

$$
g(x)=a x+b x^{3}+c x^{5}+\ldots .+d x^{m}
$$

$$
\begin{aligned}
& \mathrm{G}(\mathrm{t})=\text { Forcing Function (Periodic) } \\
& \text { Here stiff constant is a. } \\
& \mathrm{b}, \mathrm{c}, . . \mathrm{d} \text { are parameters. } \\
& m \in\{1,3,5, \ldots\} .
\end{aligned}
$$

(2) is an odd function so that one can expect oscillations which are symmetric. To the equation of motion or a harmonically forced undamped single degree of freedom oscillator, Helmholtz added the nonlinearity. The behavior of the eardrum is like an asymmetric oscillation with restoring function

$$
g(x)=a_{0} x+a_{1} x^{2}
$$

The Helmholtz equation of motion is

$$
n \frac{d^{2} x}{d t^{2}}+a_{0} x+a_{1} x^{2}
$$

Khatami et al. [1] presented general solutions for Duffing oscillations which are not linear with $5^{\text {th }}, 6^{\text {th }}$ and $7^{\text {th }}$ degree polynomial odd restoring force functions. They incorporate DTM and received fruitful results. In the case of an odd degree polynomial restoring function with symmetric oscillations of the system this method can give good results. But in the case of non-odd restoring force functions with asymmetric oscillations uncertainties are observed. In this scenario a large number of researchers made an attempt by adopting HPM in order to crack many DEqs. The primary goal of this talk is to test the acceptability HPM for Duffing Oscillators with non-odd restoring force function 
B.Mahaboob et al., International Journal of Emerging Trends in Engineering Research, 8(10), October 2020, 6684- 6688

\section{HOMOTOPY ANALYSIS METHOD}

HEM is

$\frac{d^{2} x}{d t^{2}}+x+v x^{2}=0$

$x=B, \frac{d x}{d t}=0$ at $t=0$

$v=$ nonlinearity of $\mathrm{x}$.

$\mathrm{B}=$ Amplitude of $\mathrm{x}$.

In the light of Liao [10], the HAM is applied to the NLDE (5) and a Homotopy namely d $\Omega \times[0,1] \rightarrow R$ is introduced and follows

$q\left[N(x)+L\left(x_{0}\right)\right]-\left[L\left(x_{0}\right)-L(x)\right]=0$

Here $L(x)=\frac{d^{2} x}{d t^{2}}+x$ and $N(x)=v x^{2}$

(7) is approximated with

$x=x_{0}+q x_{1}+q^{2} x_{2}+\ldots$

And $w=\lim _{q \rightarrow 1} x=x_{0}+x_{1}+x_{2}+\ldots$

Put (8) in (7) and by making comparison we can have

$L\left(x_{1}\right)+L\left(x_{0}\right)+v x_{0}^{2}=0$

$L\left(x_{2}\right)+2 v x_{0} x_{1}=0$

$L\left(x_{3}\right)+2 v\left(x_{1}^{2}+x_{0} x_{2}\right)=0$

Let the beginning approximation (5) be

$$
\begin{aligned}
& x_{0}(t)=B(\operatorname{COS}(\alpha t)) \\
& L\left(x_{0}\right)=B(\operatorname{COS} \alpha t)\left(1-\alpha^{2}\right)
\end{aligned}
$$

$\alpha(v)$ is fixed and not equal to 0 and its value at 0 is 1 .

(10) is simplified as

$\frac{d^{2} x_{1}}{d t^{2}}+x_{1}+\left(1-\alpha^{2}\right) B(\operatorname{Cos} \alpha t)+0.5 B^{2} v+0.5 B^{2} v \cos 2 \alpha t=0$

The solution of (15), gives

$x_{1}(t)=$

$\left(B+0.5 B^{2} v+0.5 B^{2} v\left(1-4 \alpha^{2}\right)^{-1}\right) \operatorname{COS} t-B \operatorname{COS} \alpha t-0.5 B^{2} v-0.5 B^{2} v\left(1-4 \alpha^{2}\right)^{-1} \operatorname{Cos} 2 \alpha t$

The secular term in (16) is eliminated by setting

$B+0.5 B^{2} v+0.5 B^{2} v\left(1-4 \alpha^{2}\right)^{-1}=0$

$\Rightarrow \alpha=0.5(S Q R T(1+B v) /(1+0.5 B v))$

$\alpha$ is 0 gives $1+B v=0 \Rightarrow B=-(v)^{-1}$

First Order Approximation to (9) is given by

$x(t)=x_{0}(t)+q x_{1}(t)$

Now

$x(t)=B \operatorname{COS} \alpha t+q\left[-B \operatorname{COS} \alpha t-0.5 B^{2} v-0.5 B^{2} v\left(1-4 \alpha^{2}\right)^{-1} \operatorname{COS} 2 \alpha t\right]$

At $q=1$

$x(t)=-0.5 B^{2} v+\left(B+0.5 B^{2} v\right) \operatorname{COS} 2 \alpha t$

For $\mathrm{B}=1 \& v=0.1(19)$ is

$x(t)=(1.05) \operatorname{COS}(1.02353 t)-0.05$
(19) gives

$\frac{d x}{d t}=\left(((1+B v) /(1+0.5 B v))(B-x)\left(B+B^{2} v+x\right)\right)^{\frac{1}{2}}$

numerically.

This is used in comparing the results with actual solution.

\section{PHASE DIAGRAM GENERATION}

To create PHASE DIAGRAM, one can see the relation as $\left(\frac{d x}{d t}\right)^{2}=\left[(B+x)+\frac{2 v}{30}\left(B^{2}+B x+x^{2}\right)\right](B-x)$

by(5) (22)

The PHASE DIAGRAM of DE (5) with boundary condition

(6) is represented by the plot of $\frac{d x}{d t}$ Vs $x$. For $\mathrm{B}=1$ and $v=0.1$ the plot of $\frac{d x}{d t}$ versus $\mathrm{x}$ created from (21) depicts a boundary which is closed. From (22) the magnitude of +ve amplitude of Non-Linear Oscillations is 1. From (22) the magnitude of -ve amplitude of Non-Linear Oscillations is -1.0717. This gives asymmetry of the PHASE DIAGRAM w.r.t $\frac{d x}{d t}$ axis, whereas it is symmetric w.r.t x-axis. (1) describes the PHASE DIAGRAM created from (22) and it shows magnitudes of +ve and -ve amplitudes which are unequal.

At $\mathrm{B}=1$ and $v=0.1$ the singularities are origin and $(-10,0)$. Origin is the singular point. $(-10,0)$ is the saddle point. In

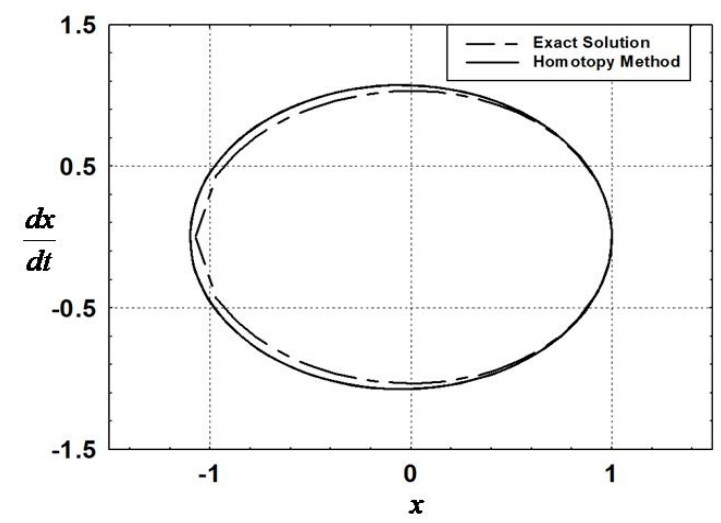

Figure 1. Helmholtz equation Vs exact solution

In order to get the periodic solution the range of amplitudes should lie in the interval $(-10,5)$. The periodic solution is impossible Out-side of this range.

In Fig.2, 3, 4, 5; the PHASE DIAGRMS are created for $B \in\{2,4,5,6\}$ and $v=\frac{1}{10}$. More over these are being compared with HOMOTOPY METHOD's solution. PHASE DIAGRAM concerning with $\mathrm{x}(0)=5$ stands for the separatrix. 
B.Mahaboob et al., International Journal of Emerging Trends in Engineering Research, 8(10), October 2020, 6684- 6688

In the case of $\mathrm{x}(0)=1$ It stands for the boundary which is closed and possessing periodicity. PHASE DIAGRAM created for $\mathrm{x}(0)=5$ by using $(21)$ is possessing close magnitudes of $+v e$ \& -ve amplitudes. Here the separatrix stands for the big amount of difference in the magnitudes of +ve \& -ve amplitudes. The HOMOTOPY solution stands exactly close to the domain at the particular boundary constraints. Fig. 6 \& 7 depict distinct PHASE DIAGRAMS from (21) and (22). HOMOTOPY METHOD is incapable to differentiate non-periodic \&periodic behavior of the solutions.

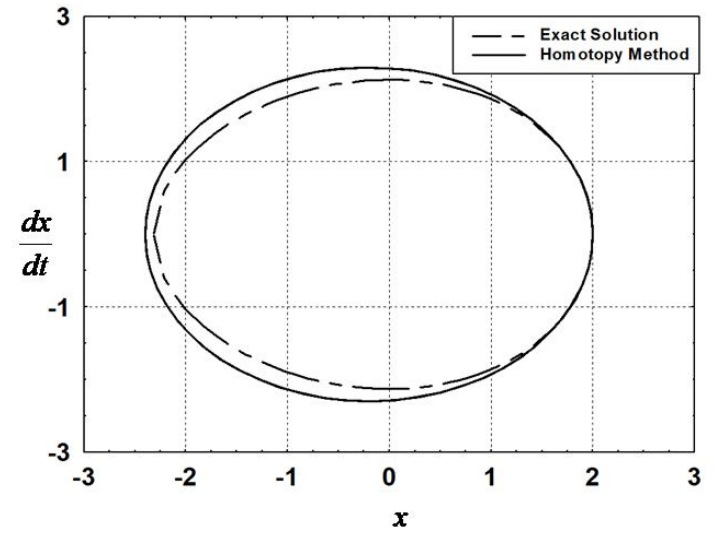

Figure 2. Comparing the PHASE DIAGRAMS for $\mathrm{x}(0)=2$

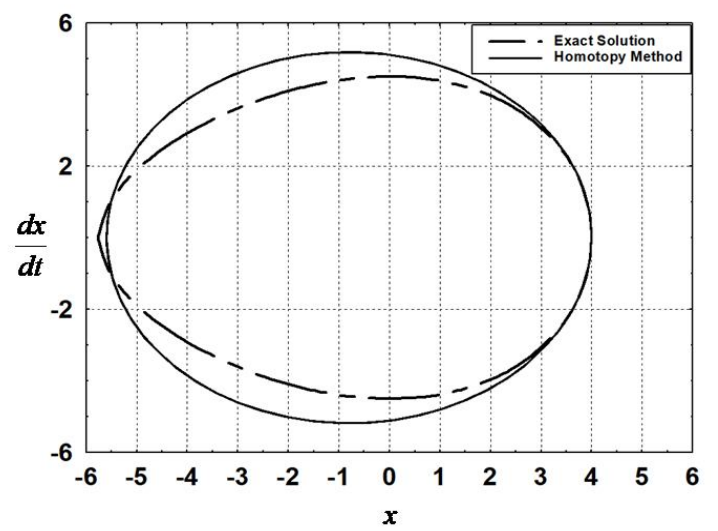

Fiure 3. Comparing the PHASE DIAGRAMS for $\mathrm{x}(0)=4$.

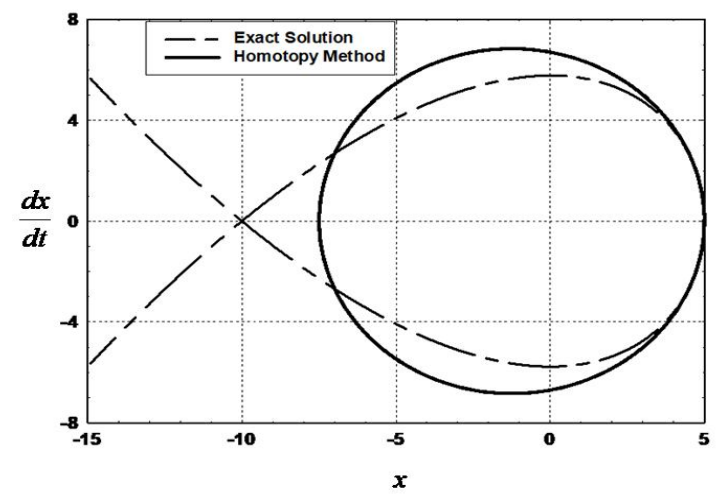

Figure 4. comparing the PHASE DIAGRAMS generated for $\mathrm{x}(0)=5$

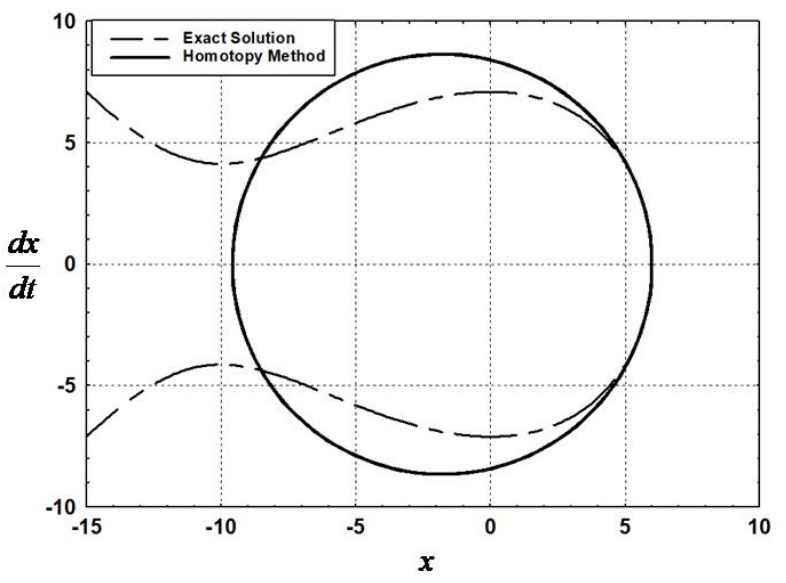

Figure 5. Comparing the PHASE DIAGRAMS for $\mathrm{x}(0)$ $=6$

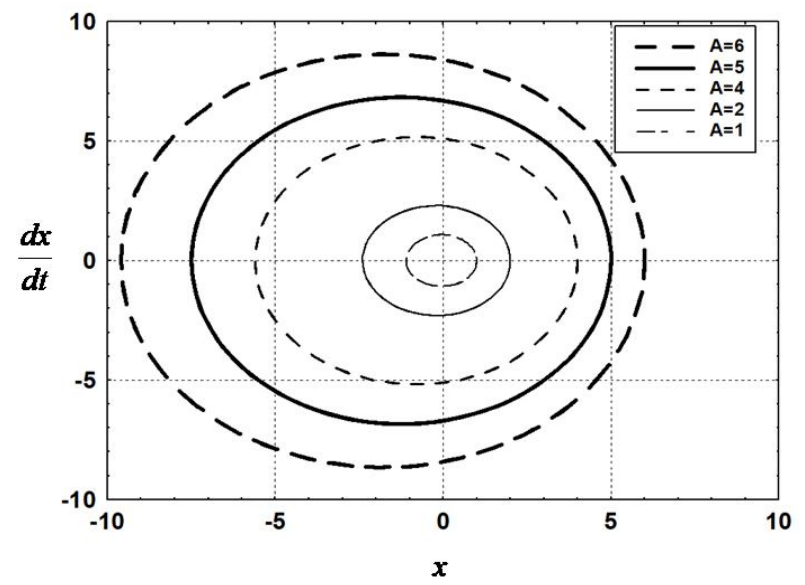

Fig. 6: PHASE DIAGRAMS generated for different amplitudes obtained by the Homotopy analysis method

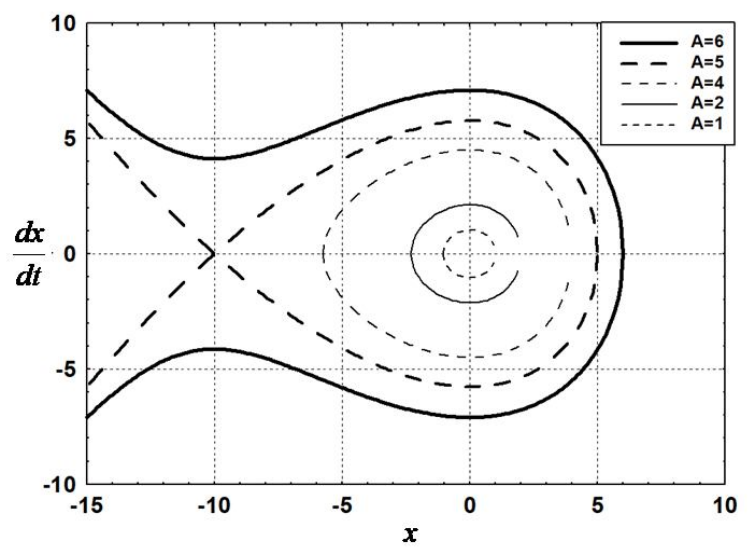

Fig.7: PHASE DIAGRAMS created out of exact solution for various amplitudes

\section{CONCLUSION}

The capability of the Homotopy Analysis Method is investigated by observing the nonlinear oscillations of Helmholtz Equation of Motion. Large discrepancy is observed 
in magnitudes of positive and negative amplitudes using the Homotopy Analysis Method in case of large amplitudes. Therefore Homotopy Analysis Method is incapable to differentiate non-periodic and periodic solutions. In the case of $\mathrm{B}$ exceeding 5 and $v=0.1$, phase plane diagram obtaining by exact solution is not closed and depicts nonperiodic nature, whereas the Homotopy Analysis Method gives the closed phase plane diagram (i.e., periodic) for all values of B. Hence, Homotopy Analysis Method is incapable to differentiate the non-periodic solutions.

\section{REFERENCES}

1. I.Khatami, E.Zahedi, M.Zahedi, Efficient Solution of Nonlinear Duffing Oscillator, Journal of Applied and Computational Mechanics, vol.6(2):219-234, 2020.

2. J.Peter Praveen, B.Nageswara Rao, On the periodic solutions of the Helmholtz equations using the modified differential transform method, MAYFEB Journal of Mathematics, Vol.2:7-16,2016.

3. J.Peter Praveen, B.Nageswara Rao, Applicability of the MDTM to obtain the periodic Solution of the Duffing type equations of motion, Asian Journal mathematics and computer Research, Vol. 18(1):19-27, 2017.

4. J.Peter Praveen, B.Nageswara Rao, Uncertainties in the Periodic Solution of a Truly Non-linear Oscillator Differential Equation Using MDTM, International Journal of Applied and Computational Mathematics, Vol.5(4) doi.org/10.1007/s40819-019-0702-3

5. M.Turkyilmazoglu, The selection of paper auxiliary parameters in the Homotopy analysis method. A case study: Uniform solution of undamped Duffing equation, Applied Mathematics, Vol.2:783-790,2017.

6. Z.Azimzadeh, A R Vahidi and E Babolian Exact solutions for nonlinear Duffing equations by He's Homotopy perturbation method, Indian Journal of Physics Vol.86(8):721-726, 2012

7. Y.Khan Y, M Akbarzade and A Kargar Coupling of homotopy and the variational approach for a conservative oscillator with strong odd-nonlinearity, Scientia Iranica vol.19(3):417-422, 2012
8. M M Zhang, Ben-Liwang, Xian-RenKong and A-Yang Xiao, Application of Homotopy perturbation method for harmonically forced Duffing systems, Applied Mechanics and Materials, Vol.110-116:.2277-2283 ,2012

9. S.Liao, Homotopy Analysis Method in Nonlinear Differential Equations, Springer, Berlin, Heidelberg, https://doi.org/10.1007/978-3-642-25132-0

10. S R Saratha, Vijeta Iyer and R Manju R , Subspace Homotopy Methods for Solving Nonlinear Equations, International Journal Recent Technology and Engineering, Vol. 7(4S):1-4 , 2018

11. Huang S,Rafiq A, Md. Rizwan Shahzad and Faisal Ali, New higher order iterative methods for solving nonlinear equations, Hacettepe $J$ Mathematics and Statistics ,Vol.47(1): 77-91,2018.

12. Wenrui Hao, A Homotopy Method for Parameter Estimation of Nonlinear Differential Equations with Multiple ,Optima, Journal Scientific Computing 74:1314-1324,2018

13. E Babolian, A R Vahidi ,Z Azimzabelu Z ,An improvement to the Homotopy perturbation method for solving nonlinear Duffing equations, Bulletin of the Malaysian Mathematical Sciences Society,Vol.41(2):1105-1117,2018.

14. Ihtisham ul Haq ,Analytical Approximate Solution of Non-Linear Problem by Homotopy Perturbation Method (HPM), Matrix Science Mathematic ,Vol.1:20-24

15. Sabri R I, Three Step Homotopy Perturbation Iteration Algorithm for Nonlinear Equations, Electronics Science Technology and Application ,Vol. 6(1):1-4 , 2019.

16. Singh R, Maurya D K, Rajoria Y K ,A Novel Approach of Homotopy Perturbation Technique to Solution of Non-Linear Fisher Equation, Interantional Journal of Applied Engineering Research 14(4):957-964,2019

17. J. Peter Praveen, R. Donthi, S.V.Prasad, B. Mahaboob, B.Venkateswarlu B., A glance on the estimation of Cobb-Douglas production functional model, AIP Conference Proceedings, 2177 (1), 020067. 2019 
18. J.Peter Praveen, B. Mahaboob, R.Donthi, S.V. Prasad, B.Venkateswarlu, On stochastic linear regression model selection, $A I P$ Conference Proceedings, 2177 (1), 020068, 2019.

19. B.Mahaboob, J.Peter Praveen, R.Donthi, S.V.Prasad, B.Venkateswarlu B., Criteria for selection of stochastic linear model selection, AIP Conference Proceedings, 2177 (1), 020041,2019

20. R.Donthi, S.V.Prasad, B.Mahaboob, J.Peter Praveen, B.Venkateswarlu. Estimation methods of nonlinear regression models, $A I P$ Conference Proceedings, 2177 (1), 020081, 2019.

21. R.Donthi, J.Peter Praveen, S.V.Prasad, B.Mahaboob, B.Venkateswarlu, Numerical techniques of nonlinear regression model estimation, AIP Conference Proceedings, 2177 (1), 020082. 2019.

22. B.Mahaboob, S.V.Prasad, J.Peter Praveen, R.Donthi, B.Venkateswarlu ,On misspecification tests for stochastic linear regression model, AIP Conference Proceedings, 2177 (1), 020039. 2019.

23. B.Mahaboob, Ajmath K.A., Venkateswarlu B., Narayana C., Praveen J.P. ,On Cobb-Douglas production function model, AIP Conference Proceedings, 2177 (1), 020040. 2019.

24. B.Mahaboob, K.A.Azmath, B.Venkateswarlu, C.Narayana, B.M.Rao, An evaluation in generalized LSE of linearized stochastic statistical model with non-spherical errors, AIP Conference Proceedings, 2177(1), 020038. 2019.

25. T.S.Rao.,G.S. Kumar,Ch.Vasavi,B.V.A. Rao., On the controllability of fuzzy difference control systems, International Journal of Civil Engineering and Technology, 8(12), PP.723-732,2017.

26. Ch.Vasavi, G.S.Kumar, T.S.Rao, B.V.A.Rao., Application of fuzzy differential equations for cooling problems, International Journal of Mechanical Engineering and Technology, 8(12), PP.712-721,2017.

27. J. Peter Praveen, B. Nageswara Rao, B.Mahaboob, M.Rajaiah, Y.Harnath, C.Narayana, On The Simulation of Beck Column through a Simple Xiong-Wang-Tabarrok Experimental Model of Centripetally Loaded Column, International Journal of Emerging Trends in Engineering

Research,vol.8(9),pp.5100-5103,2020
28. J.Peter Praveen, B.Nageswara Rao, B.Mahaboob, B.V.Appa Rao, An Application of Laplace Transform, Inverse Laplace Transform and Pade's Approximant in the Periodic Solution of Duffing Equation of Motion, International Journal of Emerging Trends in Engineering Research,vol.8(9),pp.5979-5983,2020

29. J. Peter Praveen, B. Nageswara Rao, Y. Harnath, B.V. Appa Rao, C. Narayana, and B. Mahaboob, "Existence of solution of the nonlinear differential equation in the modelling of eardrum by using homotopy perturbation method", Advances in Mathematics: Scientific Journal, Vol.9, No.7, pp.4853-4862,2020.

30. J. Peter Praveen, B. Nageswara Rao, B. Mahaboob,C.Rajaiah,Y. Harnath, , C. Narayana, Series decomposition method for asymmetric nonlinear oscillatios, Advances in Mathematics: Scientific Journal, Vol.9, No.10, pp.8069-8076,2020. 